\begin{tabular}{ccc}
\hline & International Journal of Engineering \& Technology, $7(3.20)(2018) 489-493$ \\
SPC & Website: www.sciencepubco.com/index.php/IJET \\
Research paper & Technology \\
\hline
\end{tabular}

\title{
A Design of Faceted Search Engine - a Review
}

\author{
Mohammed Najah Mahdi ${ }^{1}$, Roslan Ismail ${ }^{2}$, Abdul Rahim Ahmad ${ }^{2}$ Kavintheran Thambiratnam $^{3}$ \\ Mohammed Abdulameer Mohammed ${ }^{4}$ \\ ${ }^{1,2}$ Institute of Informatics and Computing in Energy, Universiti Tenaga Nasional, Selangor, Malaysia. \\ ${ }^{1,2}$ College of Computer Science \& Information Technology, Universiti Tenaga Nasional, Selangor, Malaysia. \\ ${ }^{3}$ Photonics Research Centre, University of Malaya, Kuala Lumpur, Malaysia. \\ najah.mahdi@uniten.edu.my \\ ${ }^{4}$ Al-Rafidain University College, Baghdad, Iraq \\ *Corresponding Author Email: mhmd@ coalrafidain.edu.iq
}

\begin{abstract}
The World Wide Web (WWW) allows the people to share information and data from large database repositories globally. The amount of information is already in the billions of databases. We need to search the information with specialize tools known generically as search engine (SE). With the huge data that needs to be handled, search engines need to retrieve meaningful information intelligently, whereby only information of interest to the searcher needs to be returned. Facets (the particular aspect or feature of something being searched) can play an important role in helping the user understand an information space better. Queries techniques within faceted search will make the search results immediate and the interaction between searcher and search engine uninterrupted and focused. They can contribute to the user's understanding of the researched terms or topics. Furthermore, they are more fun and interesting to use because users directly manipulate the search controls and the results can be displayed through choices of presentation such as text displays, transition animations, graphs etc. which bring the process closer to an experience in game playing. This paper review the design of faceted search engine.
\end{abstract}

Keywords: Information Retrieval; Search Engine; Exploratory Search; Faceted Search Engine.

\section{Introduction}

Since the advent of the WWW, people have been increasingly using the Internet as the medium to find, discover/encounter, explore, exchange, and make sense of information. Because of this, people now rely heavily on online resources to fulfill many kinds of information needs [1]. There has been a shift from only using the Web for single query-based searches to using it for more complex and exploratory search to satisfy their information needs. However, online SE and other search tools have been primarily limited to retrieve information in the form of a set of rank documents for a given query in an effective and efficient manner [2] One important aspect that is beyond the present scope of SE is analyzing the underlying search process of each user specifically performing an information search task. Although SE have evolved in smarter ways to keep track of user search history and preferences to effectively suggest queries and personalize the search results, they do not focus on the user's information search task. Thus, they fail to provide search path suggestions such as what query to execute next, which queries to exclude, which Web pages offer useful information for their task, or what information to consider as relevant to achieve the user task goal. Traditional faceted navigation styles allow one to drill down into a subject matter to find very specific documents. One limitation to this, however, is the possibility to obtain a very "narrow" view of the issue, which is recognized in Kules and Shneiderman's study [3].

\section{Motivation}

We have many SEs that gives information according to the rank retrieval (rank list) model. Generally, query response and results representing the output are arranged in a rank based on some scoring functions that combine different characteristics produced by the documents and queries. However, there are still some constraints of conventional SE which demands further study as described in the question as follows:

"Results are represented according to their rank, one of the main problems is how to rank the results returned by a SE or a combination of SEs? How do searchers think differently about their search strategy when categorized overviews are available to augment the result list and how to achieve a better accuracy in the search $[4,5]$.

\section{Related Work}

This section illustrates some relative works about Information Retrieval, Defining Relevance, Set Retrieval, Ranked Retrieval.

\subsection{Information Retrieval}

Information Retrieval (IR) is the process of searching within a document collection for a particular information need which is called a query [6]. It is finding materials (usually documents) of unstructured nature (usually text) that satisfies an information need from within large collections (usually stored on computers). 
IR typically seeks to find documents in a given collection that are about a given topic or that satisfy given information need. The topic or information need is expressed by a query, generated from the user[7]. Documents that satisfy the given query in the judgment of the user are said to be relevant. Documents that are not about the given topic are said to be non-relevant. In this section we survey related work in classifying and understanding designing search interfaces and in techniques to augment search results, but first we need to know what is considered relevance, in order to focus on improving the relevancy.

\subsection{Defining Relevance}

Probably the first notion to be defined is the notion of relevance of an IR system. That is what it means for a SE to retrieve documents that are relevant to the user [7]. The notion of relevance itself has been the source of intense debates amongst researchers often disagreeing on how to measure it $[8,9]$. However, the general consensus has been to characterize relevance either through a purely cognitive point of view or solely through a benchmarking approach. The former, which will be addressed in the later, naturally leads to the design of search user interfaces and to evaluation methods that favors user studies. In this setting, precision and recall provide a natural metric of relevance.

\subsection{Set Retrieval}

In a Boolean set retrieval model [10], a user enters a query made up of Boolean operators such as AND, NOT, OR and gets documents that match that query. The documents are returned with an unordered set and the precision, and/or recall, depends on the user's ability to write complex Boolean queries. Boolean search systems could additionally be extended with field operators to search within specific fields of the document collection. For example, a user can find terms within the title, text body, author, and other areas of the documents of interest. There has been excellent documentation of the difficulty the general public has with using Boolean search models [11]. In practice, set retrieval suffers from a clear trade-off between high precision and high recall. Because the documents returned lacked any ordering, a user can either achieve very high precision by formulating a very restrictive query or, high recall by choosing a very loose one. Users usually have to be experts in formulating complex Boolean queries in order to retrieve the most relevant set. It is important to note, however, that if the ranking of documents returned is not required due to the nature of those documents, and when the domain of interest is reserved to experts, set retrieval could be a fine approach to search For example, PubMed (www.Pubmed.com) from the United States National Library of Medicine offers an advanced search feature to help users build queries made of Boolean expressions. The user is able to create complex queries restricted to specific fields and made of AND, OR, NOT operators see Figure 1. This advanced search feature is helpful to non-expert users, considering that PubMed ranks the articles found by dates only.

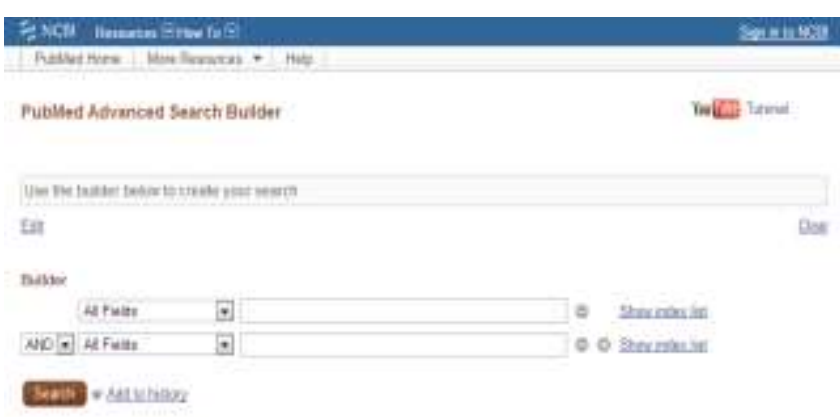

Fig. 1: The Boolean Search Interface of PubMed[12]
In order to circumvent the difficulties of the Boolean set model, an interesting compromise consists of ranking the search results. The query could remain fairly loose, but the results returned could be ranked according to some metric. In that case a user looking for books may enter some keywords related to the book and have them ordered by popularity, price or location. In the following section we will cover these called ranked retrieval models.

\subsection{Ranked Retrieval}

The first one is the vector space model approach developed by Salton, Wong, and Yang (1975)[13]. In the vector space model, each document is represented by a vector. Each index in the vector corresponds to a word or term found in the document collection. Each component of the vector is a numerical value which reflects the importance or the weight of the term in the document. The query becomes a vector which is then compared to all the other vectors document in the set. A similarity measure, usually the cosine angle between vectors, is used to match the query against the documents. The results are then ranked according to how close they are to the user's query. However, the question of properly weighting each term within the document and the collection still remains.

Another major contribution to ranked retrieval and to the vector space model is the work on $t f-i d f$ by Sparck Jones [14]. $t f-i d f$ Stands for term frequency multiply by inverse document frequency. Let us assume we have a document collection D of documents $d_{i}$ each containing terms $t_{j}$. The term frequency $t f(t, d)$ of a term $t$ within a document $d$ is the number of times $t$ appears in $d$ divided by the total number of terms in $d$.

$t f(t, d)=\frac{\left|\left\{t_{i} \in d: t_{i}=t\right\}\right|}{|d|}$

Where $\{\cdot\}$ and $|\cdot|$ denotes set definition and cardinality of a set respectively. A high term frequency indicates that a term is more representative of the document content. On the other hand, we can define the document frequency $d f(t, D)$ of a term $t$ within a document collection $D$ as the odocument frequency is the logged reciprocal of this expression.

$i d f(t, D)=\log \left(d f(t, D)^{-1}\right)=\log \frac{|D|}{\mid\left\{d_{i} \epsilon D: t \epsilon d_{i} \mid\right.}$

The inverse document frequency emphasizes rare terms over common ones. The $t f-i d f(t, d, D)$ of a term $t$ within a document $d$ in the collection $D$ is the term frequency multiply by the inverse document frequency.

$t f-i d f(t, d, D)=t f(t, d) X i d f(t, D)$

Intuitively, a term with high $t f-i d f$ is a term which is representative of the document content while not being too popular on the whole corpus. This measure will then favour frequent but rare terms in the document specific terms. The terms in the vector space model can now be weighted by $t f-i d f$ and a similarity measure can then be used in order to rank each document according to the user's query. The vector space model and $t f-i d f$ proved to be highly successful for ranking results in a set of documents which had no explicit connections with respect to each other.

However, with the advent of the WWW and hypertext collections, researchers started to develop ranking methods based on a notion of document authority. For example, a hypertext collection could be modeled as a graph with links as edges and documents as nodes. That graph can then be harnessed in order to rank documents based on a certain notion of authority, and independently of the user's query. In this respect Jon Kleinberg's HITS algorithm [15] and Larry Page and Sergey Brin's PageRank [16] were the two 
most notable measures of authority see Figure 2. The latter measure was on the basis of Google's search engine.

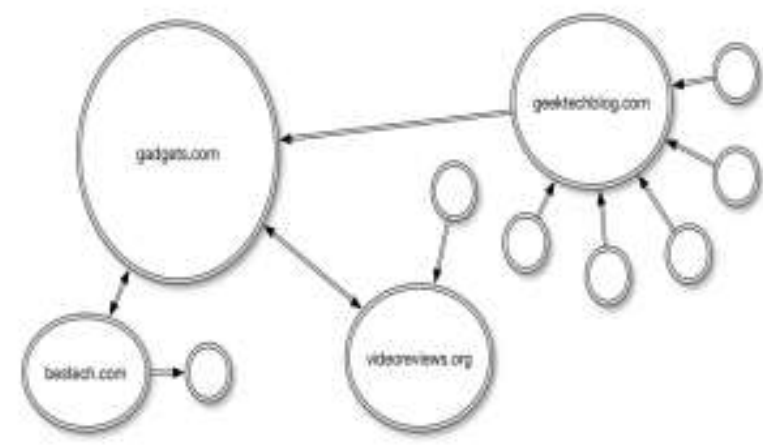

Fig. 2: PageRanks of Simple Network of Websites [17]

Today the ranking algorithms are much more complex, and PageRank, for example, is just one more signal amongst many others used. Numerous other measures of document relevance should also be noted such as F-score, Mean Average Precision (MAP) or Normalized Discounted Cumulative Gain (NDCG) [18]. Machine learning techniques could be used to train different rankers optimized on a given performance measure. The ranking models produced could even be combined or ensemble in order to achieve greater performance [19]. Furthermore, with the advent of the social web, search is now sought to be personalized to a specific user's need and profile.

\section{Exploratory Search Engine}

Current commercial SE use a process known as the query and response. The user issues a query, and receives, as a response, a set of potentially relevant documents. The process has been formalized by [20] in the lookup-based model. As shown in Figure 3, the model is comprised of four main elements. On the left hand side, the documents are processed in a summarized form understandable by the user, known as the document surrogates. On the right hand side, the user's underlying information need is reduced to a query statement. This later usually takes the form of a set of keywords together with Boolean operators. A match occurs when the document surrogates fit in the user's query. The user then investigates the surrogates, and if appropriate, delves into the documents of interest. The process may repeat itself, with the user attempting to find the right query which will yield the right set of documents.

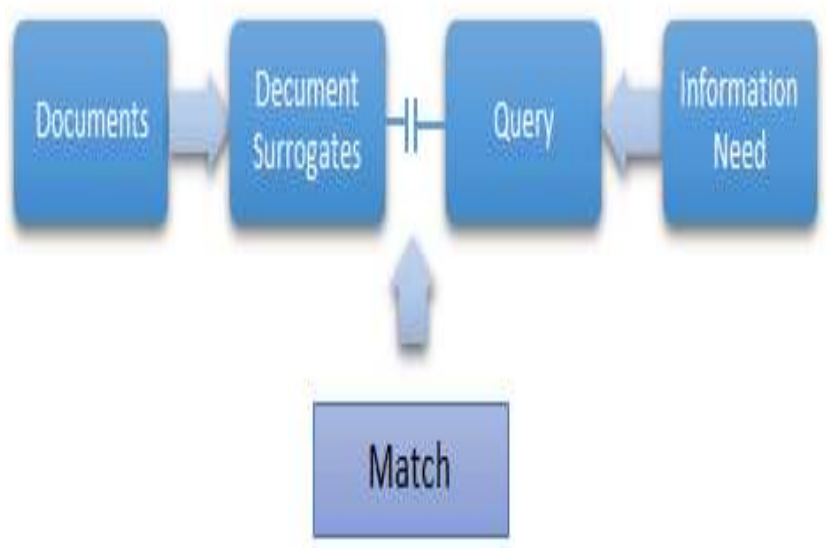

Fig. 3: The Lookup-Based Model According to Bates [20]
The lookup-based model has been identified as best suited for question answering tasks and fact finding [21]. In fact, the process must start with a carefully specified query, and should end with precise results. But the results returned, together with their potential relationships, are not intended to be further analyzed with more scrutiny.

In the look-up based model, the answer is assumed to be found in the matched documents, not necessarily in the search results themselves. The query represents a one shot summary of the user's underlying information needed. However, given today's reality of information overload, the lookup-based model appears to fall short in adequately answering the user's insatiable thirst for new information and knowledge. This has led researchers to go beyond this paradigm, and look into a new class of information seeking, known as exploratory search [24].

\section{Designing Search Interfaces}

This paper takes the perspectives of information seeking improve usability tasks likes Rocha, Zhang and González but Nielsen [25] Described five usability goals of a user interface in details we can put as, namely: learnability, efficiency, memorability, errors, and satisfaction.

Table 1: Designing Steps

\begin{tabular}{|c|c|}
\hline Usability Goals & Table 1: Designing Steps \\
\hline Learnability & $\begin{array}{c}\text { Detail } \\
\text { Relates to the facility with which first-time users } \\
\text { successfully complete initial jobs using the inter- } \\
\text { face. }\end{array}$ \\
\hline Efficiency & $\begin{array}{c}\text { Pertains to the rapidity with which users accom- } \\
\text { plish their tasks once the initial interface functions } \\
\text { are understood. }\end{array}$ \\
\hline Memorability & $\begin{array}{c}\text { Relates to the ability of a user to return to profi- } \\
\text { ciency following a period of non-use. }\end{array}$ \\
\hline Errors & $\begin{array}{c}\text { Are essential to gain understanding from the user } \\
\text { interface perspective. We intend to determine the } \\
\text { types of errors made, their frequencies, and whether } \\
\text { users can surmount them, and ultimately, become } \\
\text { successful in using the interface. }\end{array}$ \\
\hline Logically & $\begin{array}{c}\text { Errors and the other aforementioned interface as- } \\
\text { pects affect user satisfaction. }\end{array}$ \\
\hline
\end{tabular}

We need to understand clearly the manners in which users are satisfied (or dissatisfied) and to what degree. We can now explain the process of designing an interface in detail by keeping the five aforementioned usability principles in mind.

\subsection{Designing Process}

At present, web interfaces follow a user-centered approach in design. This process involves a series of steps as outlined in Figure 4 in which the user is constantly solicited [26].

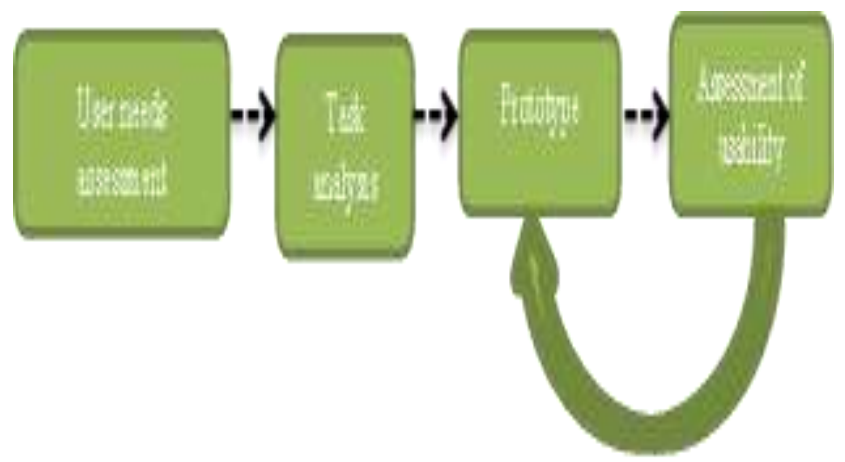

Fig. 4: User-Centered Design Approach

The series of steps of the designing process are shown in Table 2 in detail: 


\begin{tabular}{|c|c|}
\multicolumn{1}{c|}{ Table 2: Designing Steps } \\
\hline Step No & \multicolumn{1}{c|}{ Detail } \\
assessment & $\begin{array}{c}\text { Usually consists of developing a user needs assessment. } \\
\text { This may involve repeated interviews with a variety of } \\
\text { users in order to fully understand who they are and what } \\
\text { goals they have. }\end{array}$ \\
\hline Task analysis & $\begin{array}{c}\text { The designer must understand what tasks are necessary } \\
\text { for the user to achieve its goal. This step is called task } \\
\text { analysis [22] and involves that a designer chooses the } \\
\text { user goals and tasks which will be supported by the } \\
\text { interface. These steps can take the form of working } \\
\text { scenarios that typify anticipated tasks. }\end{array}$ \\
\hline $\begin{array}{c}\text { Prototype and } \\
\text { Assessment of } \\
\text { usability }\end{array}$ & $\begin{array}{c}\text { Involves the creation of a prototype that will be infor- } \\
\text { mally tested by a set of target users. This step is repeat- } \\
\text { ed by revising the prototype until the designer and the } \\
\text { users satisfy the desired usability goals. This process is } \\
\text { time-consuming and costly, and therefore, the designer } \\
\text { may select as few user participants as reasonably possi- } \\
\text { ble. The latter principle is sometimes referred to as } \\
\text { discount usability testing [23]. }\end{array}$ \\
\hline
\end{tabular}

\subsection{Small Details and Aesthetic Design}

The presented design guidelines are useful. However, attention to small details can make a significant difference between a successful and a failed interface. For example, the amount of space visually presented to a user in a query box can influence the length of the query. Users seeing a wide entry area will be encouraged to type long queries [27].

Aesthetics has an important role in user interface experience. The impression generated by the appearance of a design tends to correlate with user impression of its quality and user satisfaction [28]. However, although they provide users with a positive impression of relevance, pages with aesthetic design may actually be less useful than pages with basic design [29], In previous work [30] it was uncovered that experiential aspects such as prejudices, evoked memories, expectations can influence on how blind users experience the accessibility of a Website. Hsieh and Cheng [31] Worked on the usability of "human-computer interaction", and the users' the users' experiences to the integration of the design and aesthetic interaction principles required for the experiences of aesthetic interaction so as to make up the past shortcomings. Hotchkiss [32] interviewed a Google vice president and reported that an extensive list of details would be carefully considered in the design of the search result page. In the upper left corner, also known as the "sweet spot", Google ensures that the ads placed are not only relevant but also merge attractively within the search results.

\section{A Survey of Faceted Search}

A combination of faceted navigation and full-text search leads to a FS, as indicated in Figure 5. The structured information, or metadata, is browsed using a faceted navigation interface. The remaining unstructured data (or full text) are accessed using a simple search box. After a search is performed, the user can immediately see into which facets the results fell in. This step provides further guidance for subsequent searches and refinements. Similar to faceted navigation, FS provides guidance through the space of possible queries and their results. However, these facets generally always portray the same look and feel. They are typically represented as a hierarchical directory of choices. Interfaces that attempt to represent facets and their values with an appropriate look and feel are rare. For example, a user may want to see the location of a product on a map rather than as a list of countries or cities. The user may also be interested in relating different facets to draw insights from the data. As indicated in next section. The subsequent review is important given that FS should be implemented with a clear understanding of potential issues and challenges that may arise.

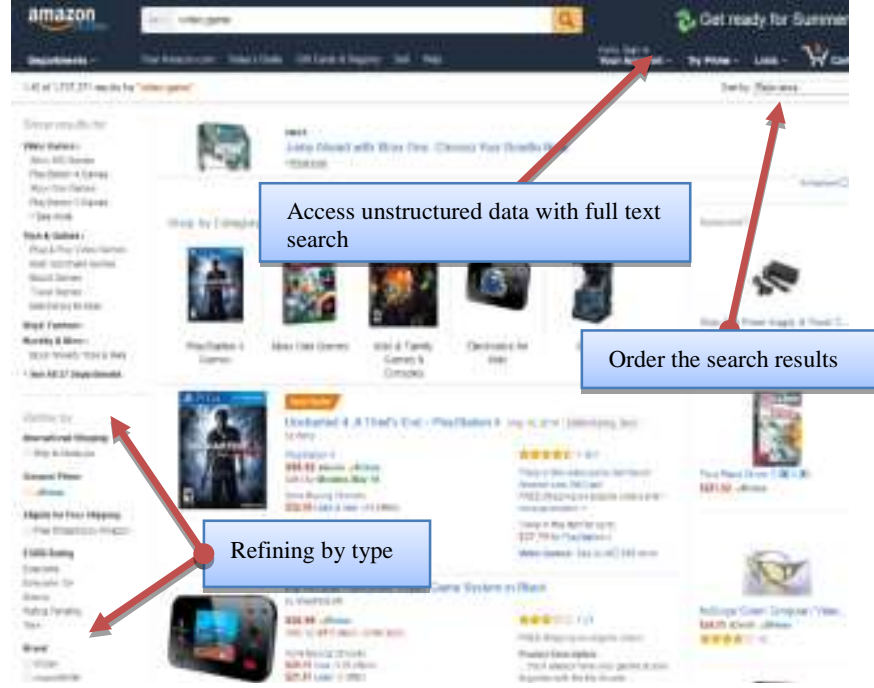

Fig. 5: Faceted Searches at Amazon.com for the Query "Video Games"

\subsection{Organizing Facets}

In this subsection, we turn our attention to organizing facets and their respective values. We merely provided common practices and recommendations, but by no means do we claim to be exhaustive, as shown in figure below:

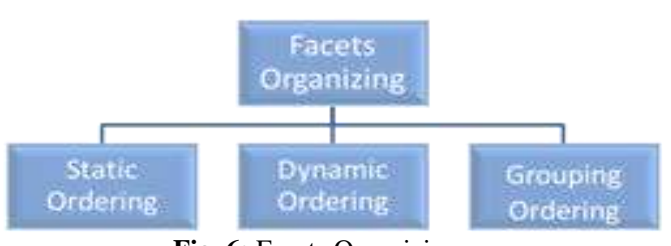

Fig. 6: Facets Organizing

The series of steps of the designing Facets Organizing process are shown in Table 3 in detail:

\begin{tabular}{|l|l|}
\hline \multicolumn{2}{|c|}{ Table 3: Facets Organizing process } \\
\hline ganizing & \multicolumn{1}{|c|}{ Details } \\
ing & $\begin{array}{l}\text { The first approach in organizing facets simply involves } \\
\text { keeping their location constant throughout the use of the } \\
\text { interface. This organization is called "static ordering", } \\
\text { and has the advantage of reinforcing the user's mental } \\
\text { model of the interface. By simply keeping each feature of } \\
\text { the interface static or constant, a user always knows } \\
\text { where to expect these features of the interface. The } \\
\text { drawback of this approach with respect to FS is that } \\
\text { several facets may not be relevant to user query, and } \\
\text { therefore, may not be useful when shown. }\end{array}$ \\
\hline $\begin{array}{l}\text { Dynamic } \\
\text { Ordering }\end{array}$ & $\begin{array}{l}\text { In contrast to the static ordering of facets, dynamic order- } \\
\text { ing places facets in a specified order based on ranking } \\
\text { algorithms that estimate the utility function of facets with } \\
\text { respect to user query. This approach is particularly useful } \\
\text { when a potentially large number of facets are possible. } \\
\text { Therefore, this approach is advantageous when only a } \\
\text { few and most relevant facets apply to user query. }\end{array}$ \\
\hline $\begin{array}{l}\text { Grouping } \\
\text { Ordering }\end{array}$ & $\begin{array}{l}\text { Another design option involves grouping related facets } \\
\text { based on some notion of similarity. A simple example } \\
\text { relates to academic journal search. Users may wish to } \\
\text { search according to authors, reviewers, name of institu- } \\
\text { tions, advisers, and so on. We can create an individual } \\
\text { facet for each of these items, but alternatively, we can } \\
\text { simply group them into a facet called "people" From this } \\
\text { grouping, we can organize the aforementioned elements } \\
\text { into sub-facets. This method is a useful means to add } \\
\text { several facets in a manner that sensibly facilitates the } \\
\text { development and refinement of user query while preserv- } \\
\text { ing static ordering. }\end{array}$ \\
\hline
\end{tabular}


Creating a hierarchy is a similar solution for presenting facets (static, dynamic, and grouping) that can achieve static ordering while simultaneously ranking the facet. Hierarchical facet values can be used in grouping even for facets that initially lack order. For example, a tree that displays the location of facet values can be formed. The designer can create and enforce any number of hierarchical values that are deemed useful.

\subsection{Exploration of Various Faceted Search Approaches}

FS allows users to explore or navigate within the document collection. However, most mainstream search systems only feature a fixed mode of interaction. For example, search results are most often depicted as a list of text with minimal interactions, such as sorting or paging. To obtain new understanding of data, allowing for multiple interaction modes is necessary. According to White and Roth [24], Exploratory Search Engine should increase user responsibility and control. This feature should include letting the user select how the data is visualized depending on the task of interest.

\section{Summary}

In this paper, we discusses exploratory search and then focus on faceted search and beyond the traditional Faceted Search interface. First, we review the rank retrieval and their Exploratory Search Engine. To promote exploration, the interface should provide instant feedback on the user's potential actions. Also provided a design of search user interfaces because the user interface of a SE forms the first and last impressions made on a user and it is a critical focal point for all users experience at every stage of the search. It is through the interface that the queries are formed and converted into informative answers. The recommendations made in this paper can be a guide for creating an interface that fosters improvements to all aspects and stages of the user search. Better interface designs assist users in articulating better queries, help them understand the results and facilitate query modifications if necessary. FS combines faceted navigation with full text search to help users to work with contents that are semi-structured whilst full text search is for non-structured contents.

\section{Acknowledgment}

This research was sponsored and supported under the Universiti Tenaga Nasional (UNITEN) internal grant no J510050783 (2018). Many thanks to the Innovation \& Research Management Center (iRMC), UNITEN who provided their assistance and expertise during the research.

\section{References}

[1] J. Curran, N. Fenton, and D. Freedman, Misunderstanding the internet: Routledge, 2016.

[2] A. Selcuk, C. Â-rencik, and E. Savas, "Private search over big data leveraging distributed file system and parallel processing," 2015.

[3] B. Kules and B. Shneiderman, "Users can change their web search tactics: Design guidelines for categorized overviews," Information Processing \& Management, vol. 44, pp. 463-484, 2008

[4] D. Bakrola and S. Gandhi, "Enhancing Web Search Results Using Aggregated Search," in Proceedings of International Conference on ICT for Sustainable Development, 2016, pp. 675-688.

[5] N. Ibrahim, A. H. Chaibi, and H. B. Ghézala, "Scientometric reranking approach to improve search results," Procedia Computer Science, vol. 112, pp. 447-456, 2017.

[6] A. N. Langville and C. D. Meyer, Google's PageRank and beyond The science of search engine rankings: Princeton University Press, 2011.

[7] C. D. Manning, P. Raghavan, and H. Schütze, "Introduction to information retrieval," ed: Cambridge University Press, 2008.
[8] S. Mizzaro, "Relevance: The whole history," JASIS, vol. 48, pp. 810-832, 1997.

[9] T. Saracevic, "Relevance: A review of the literature and a framework for thinking on the notion in information science. Part III: Behavior and effects of relevance," Journal of the American Society for Information Science and Technology, vol. 58, pp. 2126-2144, 2007.

[10] A. Singhal, "Modern information retrieval: A brief overview," IEEE Data Eng. Bull., vol. 24, pp. 35-43, 2001.

[11] D. Wolfram, A. Spink, B. J. Jansen, and T. Saracevic, "Vox populi: The public searching of the web," JASIST, vol. 52, pp. 1073-1074, 2001.

[12] "PubMed," 2017.

[13] G. Salton, A. Wong, and C.-S. Yang, "A vector space model for automatic indexing," Communications of the ACM, vol. 18, pp. 613-620, 1975

[14] K. Sparck Jones, "A statistical interpretation of term specificity and its application in retrieval," Journal of documentation, vol. 28, pp. 11-21, 1972.

[15] J. M. Kleinberg, "Authoritative sources in a hyperlinked environment," Journal of the ACM (JACM), vol. 46, pp. 604-632, 1999.

[16] S. Brin and L. Page, "The anatomy of a large-scale hypertextual web search engine," Computer networks, vol. 56, pp. 3825-3833, 1998.

[17] J. Park and S.-H. Yook, "Bayesian Inference of Natural Rankings in Incomplete Competition Networks," Scientific Reports, vol. 4, p. 6212, 08/28/online 2014.

[18] K. Järvelin and J. Kekäläinen, "Cumulated gain-based evaluation of IR techniques," ACM Transactions on Information Systems (TOIS), vol. 20, pp. 422-446, 2002.

[19] R. Caruana, A. Niculescu-Mizil, G. Crew, and A. Ksikes, "Ensemble selection from libraries of models," in Proceedings of the twenty-first international conference on Machine learning, 2004, p. 18.

[20] M. J. Bates, "The design of browsing and berrypicking techniques for the online search interface," Online review, vol. 13, pp. 407-424 1989.

[21] G. Marchionini, "Exploratory search: from finding to understanding," Communications of the ACM, vol. 49, pp. 41-46, 2006.

[22] E. Goodman, M. Kuniavsky, and A. Moed, "Observing the user experience," Burlington, Massachusetts: Morgan Kaufmann, 2012

[23] J. Nielsen, "Usability 101: Introduction to usability," ed, 2003.

[24] R. W. White and R. A. Roth, "Exploratory search: beyond the query-response paradigm (Synthesis lectures on information concepts, retrieval \& services)," Morgan and Claypool Publishers, vol. 3 , 2009.

[25] J. Nielsen, "Guerrilla HCI: Using discount usability engineering to penetrate the intimidation barrier," Cost-justifying usability, pp. 245-272, 1994

[26] S. Ben and P. Catherine, "Designing the user interface, $)^{\wedge}($ Eds.): 'Book Designing the user interface'(Reading, Mass.: Addison Wesley Longman, 1998, edn.), 2005.

[27] K. Franzen and J. Karlgren, "Verbosity and interface design," SICS Research Report, 2000.

[28] M. Hassenzahl, "The interplay of beauty, goodness, and usability in interactive products," Human-computer interaction, vol. 19, pp. 319-349, 2004

[29] T. Ben-Bassat, J. Meyer, and N. Tractinsky, "Economic and subjective measures of the perceived value of aesthetics and usability," ACM Transactions on Computer-Human Interaction (TOCHI), vol. 13 , pp. $210-234,2006$.

[30] A. Aizpurua, M. Arrue, and M. Vigo, "Prejudices, memories, expectations and confidence influence experienced accessibility on the Web," Computers in Human Behavior, vol. 51, pp. 152-160, 2015.

[31] H. C. L. Hsieh and N. C. Cheng, "A Theoretical Model for the Design of Aesthetic Interaction," in International Conference on $\mathrm{Hu}$ man-Computer Interaction, 2016, pp. 178-187.

[32] G. Hotchkiss, T. Sherman, R. Tobin, C. Bates, and K. Brown, "Search engine results: 2010," Enquiro Search Solutions, pp. 1-61, 2010. 